\title{
Tendência espaço-temporal do número de casos de dengue em Pernambuco-Brasil
}

Space-temporal analysis trend of the numbers of dengue cases in Pernambuco-Brazil

Tendencia espacio-temporal em el número de casos de dengue en Pernambuco-Brasil

Recebido: 11/05/2020 | Revisado: 12/05/2020 | Aceito: 16/05/2020 | Publicado: 25/05/2020

Jucarlos Rufino de Freitas

ORCID: https://orcid.org/0000-0002-3497-4263

Universidade Federal Rural de Pernambuco, Brasil

E-mail: jucarlos123@hotmail.com

Edgo Jackson Pinto Santiago

ORCID: https://orcid.org/0000-0003-3981-7635

Universidade Federal Rural de Pernambuco, Brasil

E-mail: edgoj@hotmail.com

Juliano Carlo Rufino de Freitas

ORCID: https://orcid.org/0000-0003-4617-4084

Universidade Federal de Campina Grande, Brasil

E-mail: julianocrufino@pq.cnpq.br

Antonio Samuel Alves da Silva

ORCID: https://orcid.org/0000-0002-8759-0036

Universidade Federal Rural de Pernambuco, Brasil

E-mail: samuelmathematical@ gmail.com

Renisson Neponuceno de Araújo Filho

ORCID: https://orcid.org/0000-0002-9747-1276

Universidade Federal do Tocantins, Brasil

E-mail: renisson@uft.edu.br

Victor Casimiro Piscoya

ORCID: https://orcid.org/0000-0003-1875-9771

Universidade Federal Rural de Pernambuco, Brasil

E-mail: victor.piscoya@ufrpe.br

Moacyr Cunha Filho

ORCID: https://orcid.org/0000-0002-3466-8143 
Universidade Federal Rural de Pernambuco, Brasil

E-mail: moacyr.cunhafo@ufrpe.br

\title{
Resumo
}

A dengue é uma doença infecciosa emergente, transmitida por mosquitos do gênero Aedes, particularmente Ae. aegypti e Ae. albopictus. O clima tropical, vasta extensão territorial e grande fluxo de pessoas no Brasil são fatores que propicia expressivas epidemias do vírus dengue (DENV). Neste contexto, a pesquisa objetivou analisar a distribuição espaço-temporal da tendência dos números de casos de dengue no estado de Pernambuco. A base de dados foi composta pelos registros epidemiológicos semanais sobre os casos de dengue de 2000 a 2018, disponibilizado através da Secretaria de Informação e Comunicação (SIC). A distribuição espacial da tendência dos quadros clínicos de dengue demonstrou concentração elevada no litoral do estado, com maior frequência de casos na Mesorregião Metropolitana do Recife (100\%), seguida pela Mata Pernambucana (71,42\%), Agreste Pernambucano (60,56\%), São Francisco Pernambucano (60,0\%) e Sertão Pernambucano (36,58\%). A espacialização da tendência permitiu delinear o cenário epidemiológico dos números de casos dengue, constituindo numa ferramenta capaz de nortear planejamentos estratégicos no controle epidêmico em Pernambuco a fim de reduzir o crescimento desenfreado da doença.

Palavras-chave: DENV; Epidemiologia; Testes; Geoprocessamento.

\begin{abstract}
Dengue is an emerging infectious disease, transmitted by mosquitoes of the genus Aedes, particularly Ae. aegypti and Ae. albopictus. The tropical climate, vast territory and large flow of people in Brazil are factors that provide expressive epidemics of dengue vírus (DENV). In this context, the research aimed to analyze the space-temporal distribution of the trend of the numbers of dengue cases in Pernambuco state. The database was composed by weeklys epidemiological records about the dengue cases from 2000 to 2018, made available through the Information and Communication Secretariat (SIC). Spatial distribution of dengue clinical pictures demonstrated a high concentration on the coast of the state, with a higher frequency of cases in the Metropolitan Region of Recife (100\%), followed by Mata Pernambucana (71,42\%), Agreste Pernambucano (60,56\%), San Francisco Pernambucano (60,0\%) e Sertao
\end{abstract} Pernambucano $(36,58 \%)$. Spatialization of the permitted trend outlines the epidemiological scenario of the numbers of dengue cases, consisting a tool capable of guiding strategic 
planning for epidemic control in Pernambuco in order to reduce the growth rampant of the disease.

Keywords: DENV; Epidemiology; Tests; Geoprocessing.

\section{Resumen}

Dengue es una enfermedad infecciosa emergente, transmitida por mosquitos del género Aedes, particularmente Ae. aegypti y Ae. albopictus El clima tropical, el vasto territorio y el gran flujo de personas en Brasil son factores que proporcionan epidemias expresivo de virus dengue (DENV). En este contexto, esta investigación tuvo como objetivo analizar la distribución espacio-temporal de la tendencia en el número de casos de dengue en el estado de Pernambuco. La base de datos consistió en registros epidemiológicos semanales sobre los casos de dengue entre 2000 y 2018, disponibles a través de la Secretaría de Información y Comunicación (SIC). La distribución espacial de la tendencia de las condiciones clínicas del dengue mostró una alta concentración en la costa del estado, con una mayor frecuencia de casos en la Mesorregión Metropolitana de Recife (100\%), seguido de la Mata Pernambucana (71,42\%), del Agreste Pernambucano (60,56 \%), del San Francisco Pernambucano (60,0\%) y del Sertão Pernambucano (36,58\%). La especialización de la tendencia permitió delinear el escenario epidemiológico del número de casos de dengue, constituyendo una herramienta capaz de guiar los planes estratégicos en el control de la epidemia en Pernambuco para reducir el crecimiento desenfrenado de la enfermedad.

Palabras clave: DENV; Epidemiología; Pruebas; Geoprocesamiento.

\section{Introdução}

Segundo Ferreira, Chiaravalloti Neto e Mondini, (2018), 390 milhões de pessoas são infectadas anualmente, acarretando cerca de 500 mil internações e 20 mil óbitos. No Brasil, especificamente no estado de Pernambuco, foram notificados, em 2015, o maior número de casos de dengue na história, ou seja cerca de 102.721 casos, 26 casos graves, 74 casos com sinal de alarme, resultando em 23 óbitos.

Estudo extensivo realizado por Teixeira et al. (2013), demonstram um aumento geral na distribuição e gravidade da dengue no Brasil, no período de 2000 a 2010, em relação a década passada, em que a co-circulação de vários sorotipos de DENV e alta endemicidade da dengue podem ser responsáveis pela aumento da ocorrência de formas graves de dengue e aumento do número de hospitalizações relacionadas à dengue. Diante deste cenário, faz-se 
necessário desenvolver pesquisas capazes de averiguar a tendência espaço temporal do crescimento/decrescimento de epidemias em um país, região ou estado, a fim de fornecer subsídios para as agências reguladoras de saúde estruturar suas estratégias de ação.

O mosquito do Aedes aegypti desenvolve-se melhor em países tropicais e subtropicais, além disso, encontra-se ativo durante o dia, e seus ovos são resistentes e sobrevivem por vários meses na ausência de água (Farnesi et al., 2019). Embora, vacinas sejam comercializadas no tratamento dos quatro sorotipos antigenicamente distintos (DENV-1 a 4) (Campos et al., 2018), isto não soluciona o problema da proliferação deste vetor e estudos descrevem um novo sorotipo, o DENV-5 (de Sousa et al., 2020), sendo, portanto, mais eficiente promover campanhas de conscientização pública através de dados parametrizados e que norteiem as ações governamentais, além de investir no desenvolvimento de compostos que atuem como larvicidas para erradicação deste vetor (Menezess et al., 2015).

O processo de urbanização, sem planejamento, implicou no surgimento de diversos problemas relacionados às epidemias promovidas por arboviroses (Wilke; Wilk-da-Silva; Marrelli, 2017). No geral, essas arboviroses são transmitidas por diferentes espécies de mosquitos, destacando-se o Aedes aegypti, principal vetor de doenças graves, a citar: dengue, febre amarela, Zika Vírus e Chikungunya (Kraemer et al., 2019).

Vale ressaltar que a proliferação do Aedes aegypti está intrinsecamente ligada as condições sócio demográficas, aspectos biológicos, descarte inapropriado do lixo e acúmulo de água em recipientes inadequados. Além disso, as variáveis climatológicas contribuem para o aparecimento de novas doenças, possibilitando o surgimento de doenças reemergentes (Barcellos et al., 2009; Valle; Pimenta; Cunha, 2015; Marques; Siqueira; Portugal, 2020).

O trabalho visa, através de técnicas estatísticas não paramétricas, identificar tendências de aumento/diminuição bem como mudanças bruscas nas médias anuais de casos de dengue no estado de Pernambuco. Os resultados obtidos podem ser utilizados pelo governo do estado para elaborar estratégias e mitigar o número de casos e mortes para região em estudo.

\section{Metodologia}

O conjunto de informações utilizadas para o desenvolvimento do trabalho remetem ao número de casos de dengue semanais no estado de Pernambuco, disponibilizados pela Secretaria de Informação e Comunicação (SIC), no período de janeiro 2000 a dezembro de 2018, compreendendo os 185 municípios do estado. 
A tendência na Série Temporal (ST) representa o comportamento da série ao longo do tempo, refletindo na evolução global no sentido do crescimento ou decrescimento do nível da série. No estudo foi utilizado pelo teste sequencial de Mann-Kendall (SMK), proposto por Sneyres (1990).

O teste SMK é um teste não paramétrico, deriva-se de um teste de correlação de classificação para dois grupos de observações, proposto por Kendall (1995), no qual, verifica se as observações são independentes e identicamente distribuídas, cuja as hipóteses são definidas por:

$H_{0}$ : As observações da série não possuem tendências

$H_{1}$ : As observações da série possuem tendência monotônica no tempo

Seja $Z_{t}=\left\{z_{t} \in R \mid t=1,2, \ldots, N\right\}$ as observações de uma série temporal, em que $t$ é um índice cronológico e $N$ é a quantidade de observações, o procedimento consiste em realizar a soma de $t_{n}=\sum_{i=1}^{N} m_{i}$ do número de termos $m_{i}$ da série, relativo ao valor $\mathrm{Z}_{\mathrm{t}}$ cujos termos precedentes $(j<i)$ são inferiores ao mesmo $\left(Z_{i}<Z_{j}\right)$ (Pinheiro; Graciano; Severo, 2013). À medida que o tamanho $N$ da amostra aumenta, sob a hipótese nula de não existência de tendência, $t_{n}$ apresentará uma distribuição normal com média e variância, respectivamente, por:

$E\left(t_{n}\right)=\frac{N(N-1)}{4}$

$\operatorname{Var}\left(t_{n}\right)=\frac{N(N-1)(2 N+5)}{72}$

Sendo assim, sob $H_{0}$, utilizando o teste bilateral, a estatística do teste $U\left(t_{n}\right)$ é definida por:

$U\left(t_{n}\right)=\frac{t_{n}-E\left(t_{n}\right)}{\sqrt{\operatorname{Var}\left(t_{n}\right)}}$

em que rejeita-se $H_{0}$ para grandes valores de $U\left(t_{n}\right)$. Outra alternativa consiste em calcular o valor de $\alpha^{*}$ por meio de uma tabela de distribuição normal padronizada, tal que: rejeita $H_{0}$ se $\alpha^{*}=P\left(|U|>\left|U\left(t_{n}\right)\right|\right)<\alpha$, sendo $\alpha$ o nível de significância $(\alpha=0.05)$. Caso $H_{0}$ seja rejeitada, o sinal da estatística do teste $U\left(t_{n}\right)$ indicará tendência positiva $U\left(t_{n}\right)>0 \mathrm{ou}$ negativa $U\left(t_{n}\right)<0$. 
Assim, a estatística do teste $U\left(t_{n}\right)$ é calculada no sentido direito da série, isto é, partido de $1 \leq t \leq N$. De modo análogo, aplica-se o mesmo princípio no sentido esquerdo da série, ou seja, $N \leq t \leq 1$, definindo assim, a estatística inversa $U^{*}\left(t_{n}\right)$. Se houver ponto de intersecção entre as curvas $U\left(t_{n}\right)$ e $U^{*}\left(t_{n}\right)$, detecta-se uma possível mudança de tendência na série analisada. No entanto, o ponto de mudança só é significativo se as intersecções das curvas ocorrem dentro dos valores críticos $-1,96<U\left(t_{n}\right)<1,96$.

Em seguida, aplica-se o teste de homogeneidade de Pettitt (PETT) para determinar o ponto (ou ano) aproximado que inicia a quebra da tendência (Pettit, 1979; Yu; Yang; Kuo, 2006). O teste PETT trata-se de um teste não paramétrico baseado no teste Mann-Whitney que verifica se duas amostras $Z_{1}, Z_{2}, \ldots, Z_{t}$ e $Z_{t+1}, Z_{t+2}, \ldots, Z_{T}$ provêm da mesma população (Nachar, 2008).

$\mathrm{Na}$ etapa inicial do teste, calcula-se a contagem do número de vezes que um membro da primeira amostra é maior que um membro da segunda amostra. Desse modo, a estatística do teste $U_{t_{t} T}$ é definida por:

$U_{t, T}=U_{t-1, T}+\sum_{i=1}^{T} \operatorname{sgn}\left(Z_{i}-Z_{j}\right)$, para $i=2, \ldots, T$

em que $\operatorname{sgn}(z)$ representa a função sinal e assume valores:

$\operatorname{sgn}\left(Z_{i}-Z_{j}\right)=\left\{\begin{array}{r}1, Z_{i}>Z_{j} \\ 0, Z_{i}=Z_{j} \\ -1, Z_{i}<Z_{j}\end{array}\right.$

Posteriormente, obtêm-se a estatística $K(t)=\max _{1 \leq t \leq T}\left|U_{t_{2} T}\right|$ pelo teste PETT, onde localiza o ponto de mudança brusca na série temporal cuja a significância pode ser avaliada, de acordo com a equação (5):

$p \cong 2 \cdot \exp \left(\frac{\left.-6 \cdot K(t)^{2}\right)}{T^{3}-T^{2}}\right)$

em que $K(t)$ representa o valor máximo absoluto de $U_{t, T}$ e $T$ o tamanho da série. O ponto de mudança (ou descontinuidade) da série é verificado quando o valor de $t$ ocorre para o valor máximo de $K_{\text {erit. }}$

$K_{\text {erit. }}= \pm \sqrt{\frac{\ln \left(\frac{p}{2}\right)\left(T^{3}-T^{2}\right)}{6}}$ 


\section{Resultados e Discussão}

O estado de Pernambuco é composto por 185 municípios com uma população de 9.557.071 milhões de pessoas, segundo os dados de 2019 do Instituto Brasileiro de Geografia e Estatística (IBGE). Os testes de SMK e de PETT foram empregados para todos os municípios do estado. Inicialmente, o estudo foi realizado para os cinco municípios mais populosos das cinco mesorregiões do estado de Pernambuco: Serra Talhada (Sertão Pernambucano, população de 86.350 habitantes), Petrolina (São Francisco Pernambucano, população de 349.145 habitantes), Caruaru (Agreste Pernambucano, população de 361.118 habitantes), Vitória do Santo Antão (Mata Pernambucana, população de 138.757 habitantes) e Recife (Metropolitana do Recife, população de 1.645.727 habitantes), em seguida, para a totalidade do estado, em forma de gráfico, com o intuito de interpretar as tendências das ST, utilizando-se os testes SMK e PETT.

Com a finalidade de estabelecer um critério que expresse os resultados dos testes SMK e PETT, utilizou-se a seguinte convenção: os sinais $(++)$ e $(--)$, para tendência positiva e negativa, cujo primeiro sinal correspondente ao teste SMK e o segundo ao teste PETT respectivamente, tornando significativos se o p-valor for abaixo de 5\%. Os sinais (? -), (? +) e (? ?) foram adotados para indicar ausência de tendência na série avaliada, independente do segundo teste (? -) e (?+).

A Figura 1 apresenta os testes de SMK e de PETT aplicados para o número de casos semanais de dengue no município de Caruaru/PE. Nos gráficos dessas figuras as linhas pontilhadas horizontais indicam os intervalos de confiança para o teste de Mann-Kendall ao nível de significância de 5\%, observa-se uma tendência decrescente (- -) do número de casos. Esse resultado está relacionado ao teste SMK (Figura 1a), em que houve cruzamentos das curvas $U\left(t_{n}\right)$ e $U^{*}\left(t_{n}\right)$ entre os intervalos de confiança estabelecido, tornando-se significativo na 52a semana epidemiológica (SE) de 2004 e o ponto de mudança brusca de $K(t)$ pelo teste de PETT (Figura 1b), ocorreu ao cruzar o limite de 5\% com máximo inferido em 2004. Confirmando assim, o registro de tendência negativa (- -) para ambos os testes a partir de 52 ${ }^{\text {a }}$ SE de 2004 nessa localidade. 
(CC BY 4.0) | ISSN 2525-3409 | DOI: http://dx.doi.org/10.33448/rsd-v9i7.4427

Figura 1. Testes não paramétricos (a) SMK e (b) PETT para o número de casos semanais de dengue no município de Caruaru/PE, no período de janeiro de 2000 a dezembro 2018.
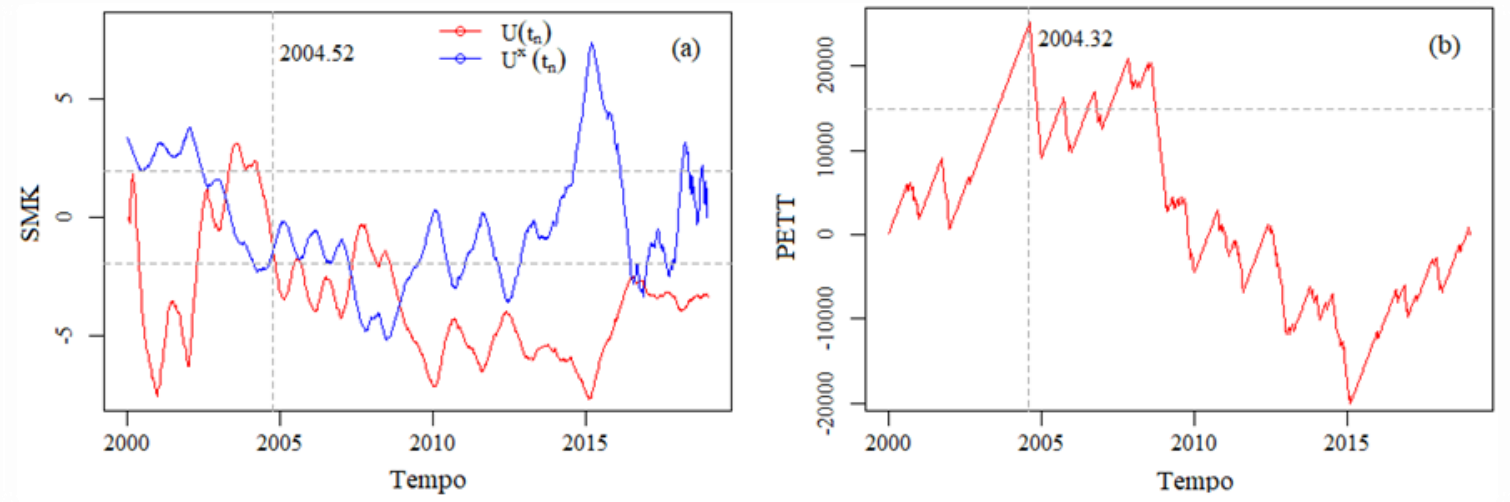

Fonte: Elaborada pelos Autores (2020).

Ao analisar o número de casos de dengue no município de Petrolina/PE, verifica-se o registro de tendência positiva $(++)$ a partir da $30^{\mathrm{a}} \mathrm{SE}$ de 2002 (Figura 2a) e o ponto de mudança brusca de $K(t)$, ocorreu ao cruzar o limite crítico estabelecido de $5 \%$ na primeira SE de 2010 (Figura 2b). Segundo Rei, Abrahão e Moura (2018), a proliferação de doenças nessa região está associada aos impactos ambientais provocados no Rio São Francisco, em decorrência do desenvolvimento acelerado das cidades de Petrolina e Juazeiro.

Figura 2. Testes não paramétricos (a) SMK e (b) PETT para o número de casos semanais de dengue no município de Petrolina/PE, no período de janeiro de 2000 a dezembro 2018.
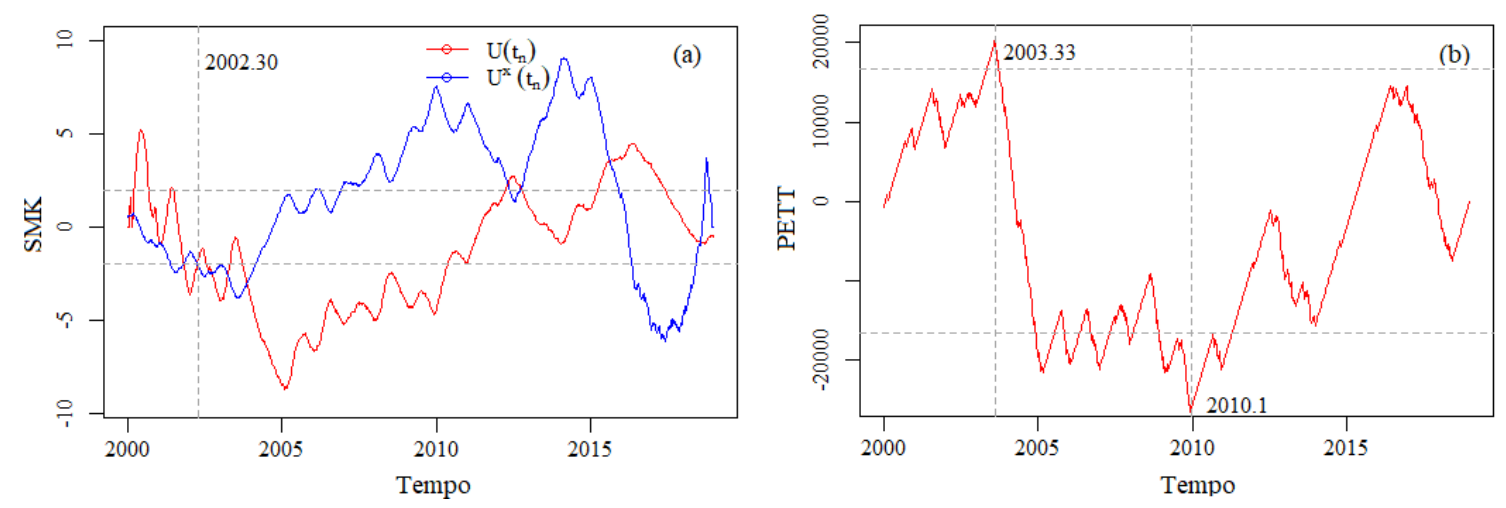

Fonte: Elaborada pelos Autores (2020).

Para o número de casos de dengue no município de Recife, nenhuma tendência foi confirmada, visto que o cruzamento das curvas estatísticas no teste SMK ocorreu fora dos intervalos de confiança de 5\% (Figura 3a), e a curva estatística do teste PETT (Figura 3b) ter 
(CC BY 4.0) | ISSN 2525-3409 | DOI: http://dx.doi.org/10.33448/rsd-v9i7.4427

cruzado o nível de significância e registrado valor mínimo em 2005. A ausência de tendência é notória, pois o município registra casos de dengue recorrente com índices que demonstram situação constante de risco para a ocorrência de surto ou encontra-se em situação de alerta.

Figura 3. Testes não paramétricos (a) SMK e (b) PETT para o número de casos semanais de dengue no município de Recife/PE, no período de janeiro de 2000 a dezembro 2018.
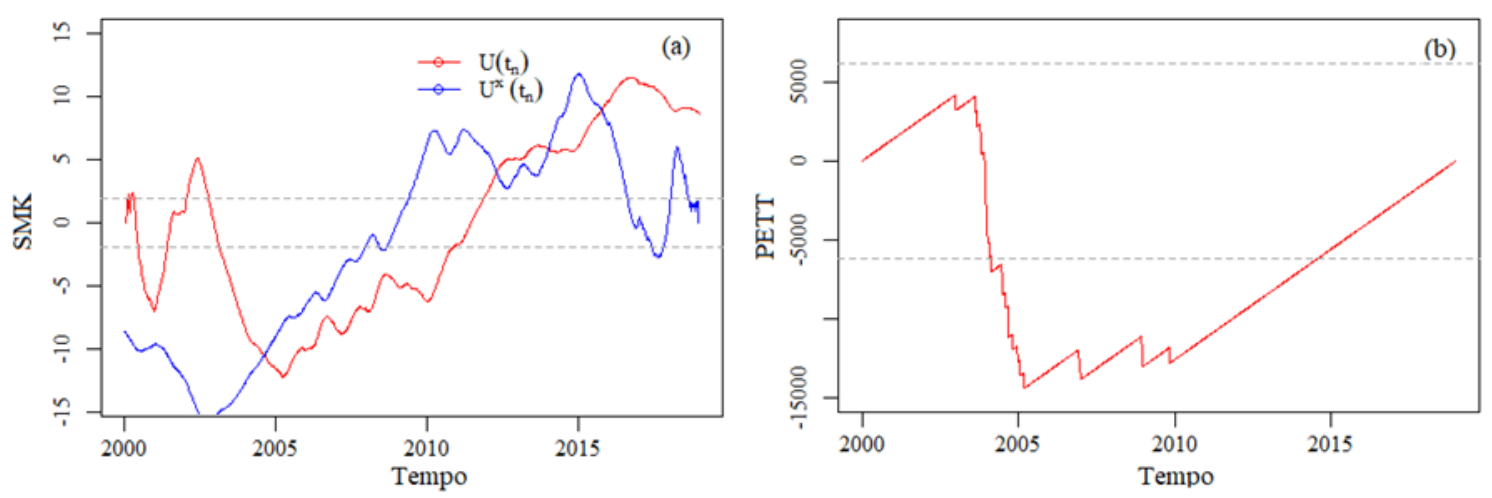

Fonte: Elaborada pelos Autores (2020).

Entretanto, para o município de Serra Talhada/PE foi inferida tendência negativa (- -) no número de casos de dengue a partir da primeira SE de 2018, pois o cruzamento registrado pelas curvas estatísticas no teste SMK (Figura 4a), representando o indício de tendência na ST. Visando confirmar essa tendência, aplicou-se o teste PETT (Figura 4b) e verificou-se que a curva ultrapassou o nível de significância de 5\%, com máximo registrado em $17^{\mathrm{a}} \mathrm{SE}$ de 2017, conferindo um elevado grau de significância para essa tendência para essa data.

Figura 4. Testes não paramétricos (a) SMK e (b) PETT para o número de casos semanais de dengue no município de Serra Talhada/PE, no período de janeiro de 2000 a dezembro 2018.
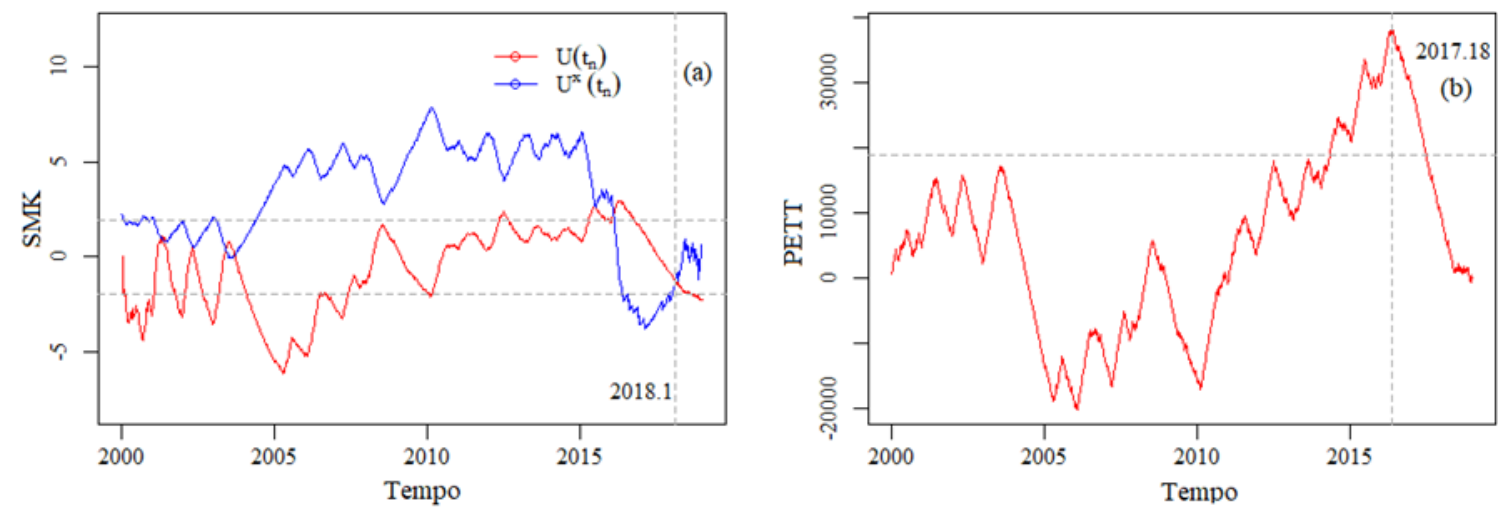

Fonte: Elaborada pelos Autores (2020). 
Para o município de Vitória de Santo Antão/PE foi possível verificar que o teste SMK (Figura 5a) acusou vários cruzamentos das curvas estatísticas $U\left(t_{n}\right)$ e $U^{*}\left(t_{n}\right)$ entre os intervalos de confiança, dando indícios de haver tendência nessa variável, porém numa data indefinida, tornando necessário o uso do teste PETT. Observa-se, na Figura 5b, que o ponto de mudança brusca de $K(t)$, ocorreu ao cruzar o limite crítico estabelecido de $5 \%$ na primeira SE de 2015, confirmando, desta maneira, tendência de crescimento $(++)$ para o número de casos de dengue no período estudado.

Figura 5. Testes não paramétricos (a) SMK e (b) PETT para o número de casos semanais de dengue no município de Vitória de Santo Antão/PE, no período de janeiro de 2000 a dezembro 2018.
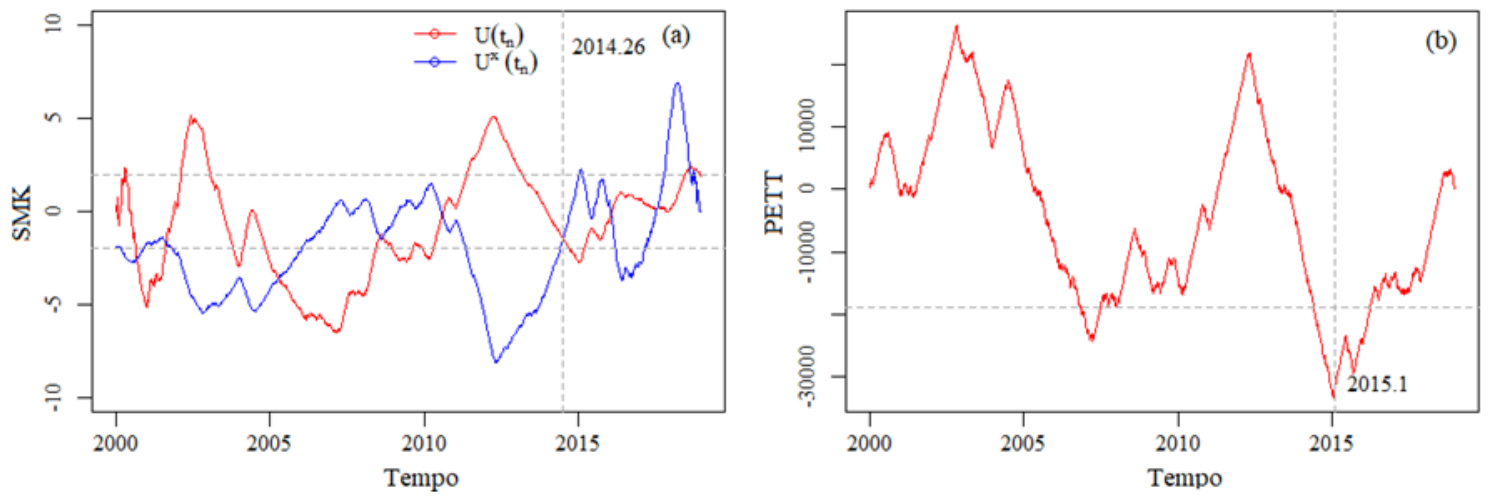

Fonte: Elaborada pelos Autores (2020).

Por meio da análise gráfica dos testes não paramétrico, SMK e PETT, para os cinco municípios do estado de Pernambuco, constatou-se que Caruaru, Petrolina, Serra Talhada e Vitória do Santo Antão apresentou existência da tendência nas STs, enquanto que Recife não apresentou existência de tendência nas STs. Para os demais municípios foram realizados os testes SMK e PETT e o resultados apresentados em escala espacial. A Figura 6 apresenta o resumo destes resultados obtidos pelos testes do número de casos semanais de dengue, para o período de janeiro de 2000 a dezembro de 2018. Verifica-se pelos testes que os municípios do estado de Pernambuco apresentaram uma tendência estatisticamente significativa (pvalor $<0.05$ ) de crescimento (verde), decrescimento (vermelho) ou ausência de tendência (amarelo).

Analisando a espacialização, nota-se que os municípios próximos ao litoral (Região Metropolitana do Recife) apresentaram uma tendência positiva para $100 \%$ de seus municípios, entre eles 46,67\% apresentou ausência pelo teste SMK (?+), mas com indícios de 
aumento para o teste de PETT, conforme a Tabela 1. Este aumento possivelmente estar associado a fatores climatológicos, pois os municípios da Mesorregião Metropolitana do Recife sofrem influências da Zona de Convergência Intertropical (ZCIT), El Ninõ, La Niña e eventos extremos que influenciam na alteração do ecossistema e de ciclo biogeoquímico, que podem acelerar a propagação de doenças infecciosas (Collischonn; Dubreuil; Mendonça, 2018). Resultado corroborado por Ribeiro et al. (2016) e Monteiro et al. (2009), demonstram que períodos chuvosos aumentam o número de casos de dengue, além da precipitação, a temperatura elevada apresenta uma correlação positiva na transmissão de dengue.

Tabela 1. Resultados dos percentuais de tendências significativas para cada mesorregião do estado de Pernambuco, no período de janeiro de 2000 a dezembro de 2018, por meio da aplicação dos testes não paramétricos SMK e PETT.

\begin{tabular}{lccccc}
\hline \multirow{2}{*}{ Mesorregião } & \multicolumn{5}{c}{ Tendência } \\
\cline { 2 - 6 } & $(++)$ & $(?+)$ & $(--)$ & $(?-)$ & $(? ?)$ \\
\hline Metropolitana do Recife & $53,33 \%$ & $46,67 \%$ & $0,0 \%$ & $0,0 \%$ & $0,0 \%$ \\
Mata Pernambucana & $30,95 \%$ & $40,47 \%$ & 16,67 & $9,52 \%$ & $2,38 \%$ \\
Agreste Pernambucano & $33,80 \%$ & $26,76 \%$ & $25,35 \%$ & $14,08 \%$ & $0,0 \%$ \\
São Francisco Pernambucano & $33,33 \%$ & $26,67 \%$ & $26,67 \%$ & $13,33 \%$ & $0,0 \%$ \\
Sertão Pernambucano & $21,95 \%$ & $14,63 \%$ & $46,34 \%$ & $12,19 \%$ & $4,87 \%$ \\
\hline
\end{tabular}

Fonte: Elaborada pelos Autores (2020).

Semelhantemente a Metropolitana do Recife, a Mata Pernambucana é endêmica para dengue e apresenta uma tendência positiva para 71,42\% de seus municípios, entre eles $40,47 \%$ apresentou ausência pelo teste SMK (? +), mas com indícios de aumento para o teste de PETT, no período estudado. A Mata Pernambucana tem como principais características as altas temperaturas, em média $25^{\circ}$, e alto índice de pluviosidade no outono e inverno (Silva; Nóbrega, 2012) influenciada pela proximidade com o Oceano Atlântico, favorecendo a expansão de doenças infecciosa. De modo análogo, o Agreste Pernambucano apresentou a mesmas características que a Mata Pernambucana com tendência positiva para $60,56 \%$ de 
(CC BY 4.0) | ISSN 2525-3409 | DOI: http://dx.doi.org/10.33448/rsd-v9i7.4427

seus municípios, entre eles 26,76\% apresentou ausência pelo teste SMK (? +), mas com indícios de aumento para o teste de PETT.

Ressalta-se que, os municípios do Agreste, Mata e Metropolitana do Recife são compostos por vegetação litorânea e Mata Atlântica sendo capaz de influenciar negativamente o desenvolvimento do vetor (Lins; Candeias, 2018), contudo, o desmatamento na região contribuiu para a expansão dos mosquitos, já que há perda e fragmentação do seu habitat (Mayer; Tesh; Vasilakis, 2017). Um estudo extensivo realizado por Moura et al. (2014), demonstra que desmatamento da Mata Atlântica apresentou relação positiva estatisticamente significativa com a incidência da doença no Estado do Rio de Janeiro, nos anos de 2001 a 2008.

Figura 6. Espacialização da distribuição de tendências do número de casos semanais de dengue para o estado de Pernambuco, no período de janeiro de 2000 a dezembro de 2018, por meio da aplicação dos testes não paramétricos SMK e PETT.

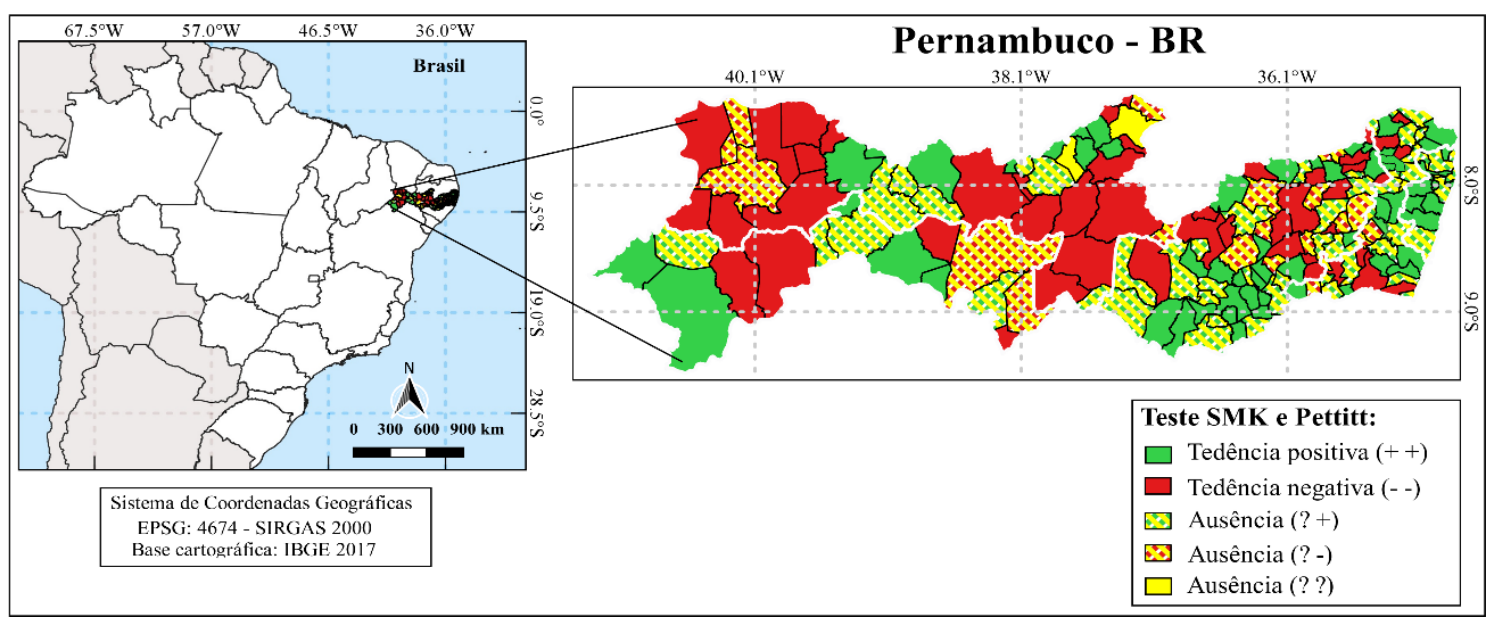

Fonte: Elaborada pelos Autores (2020).

No entanto, contata-se que o número de casos de dengue diminui com o distanciamento do litoral, ou seja, os municípios localizados no Sertão Pernambucano apresentaram menores amplitudes em relação as demais Mesorregiões com tendência negativa para 58,53\% de seus municípios, entre eles $12,19 \%$ apresentaram ausência pelo teste SMK (? -), mas com indícios de redução para o teste de PETT. Esta redução pode estar relacionada com clima semiárido da região, ocasionado assim, a falta de chuvas que propiciam a não eclosão dos ovos do mosquito (Baracho et al., 2014).

Já a Mesorregião do São Francisco Pernambucano apresentou comportamentos variados com tendências de crescimento e decrescimento para $33,33 \%$ e $26,67 \%$ de seus 
municípios. Também, observa-se que os municípios que faz divisa com a Bahia apresentaram tendência positiva, com exceção de Lagoa Grande, Santa Maria da Boa Vista e Jatobá, este aumento pode estar associado ao Rio São Francisco que atravessa o estado da Bahia, fazendo sua divisa ao norte com Pernambuco, sendo este um possível fator de disseminação na região. Além disso, analisando o mapa geográfico da região foi verificado que existem três Barragens: Sobradinho, Itaparica e Paulo Afonso próximas ao Norte de Pernambuco que podem influenciar a distribuição dos vetores e necessariamente dos vírus (Ebi; Nealson, 2016).

\section{Considerações Finais}

A análise espaço-temporal da tendência em Pernambuco apontam possíveis causas de mudanças observadas nas STs, tendo em vista a complexidade em associar essas alterações devido a eventos extremos, como o El Ninõ, La Niña, desmatamentos, queimadas, grande fluxo de pessoas, favorecimento do clima tropical e vasta extensão territorial, entre outros fatores que influenciam na alteração do ecossistema e de ciclo biogeoquímico, que podem acelerar a propagação de doenças infecciosas.

No mais, os resultados obtidos pelo geoprocessamento demonstram áreas com indícios de tendência e mudanças bruscas nas médias anuais fornecendo subsídios para as agências reguladoras de endemias e epidemias do estado de PE montarem suas estratégias de ação.

\section{Agradecimentos}

O presente trabalho foi realizado com apoio da Coordenação de Aperfeiçoamento de Pessoal de Nível Superior - Brasil (CAPES) - Código de Financiamento 001. À Universidade Federal Rural de Pernambuco (UFRPE); ao Programa de Pós-Graduação em Biometria e Estatística Aplicada (PPGBEA) e a Secretaria de Informação e Comunicação (SIC).

\section{Referências}

Baracho, R. C. M., Ismael Filho, A., Gonçalves, A., Nunes, S. D. T. S., \& Borges, P. D. F. (2014). A influência climática na proliferação da dengue na cidade de Areia, Paraíba. Revista Gaia Scientia, 8(1), 65-73. 
Barcellos, C., Monteiro, A. M. V., Corvalán, C., Gurgel, H. C., Carvalho, M. S., Artaxo, P., Hacon, S., \& Ragoni, V. (2009). Mudanças climáticas e ambientais e as doenças infecciosas: cenários e incertezas para o Brasil. Epidemiologia e Serviços de Saúde, 18(3), 285-304. http://dx.doi.org/10.5123/S1679-49742009000300011.

Campos, J. M., Oliveira, D. M. D., Freitas, E. J. D. A., \& Neto, A. C. (2018). Arboviroses de importância epidemiológica no Brasil. Revista de Ciências da Saúde Básica e Aplicada, 1(1), 36-48.

Collischonn, E., Dubreuil, V., \& Mendonça, F. D. A. (2018). Relações entre o clima e saúde: o caso da dengue no Rio Grande do Sul no período de 2007 a 2017. Confins. Revista Francobrasilera de Geografia, (37), 1-57. https://doi.org/10.4000/confins.15431.

De Sousa, M. M. F., De Melo, B. D. O., Costa, A. K. S., Nunes, J. P. P., Monteiro, S. G., De Carvalho, A. C. P., Pinto, C. M. F. S., Cosme, L. M. S. S., TurRI, R. J. G., Texeira, M. M., \& Bomfim, M. R. Q. (2020). Detection and differentiation of dengue virus serotypes by one-step multiplex reverse transcription PCR assays. Brazilian Journal of Development, 6(1), 227-246. https://doi.org/10.34117/bjdv6n1-016.

Ebi, K. L., \& Nealon, J. (2016). Dengue in a changing climate. Environmental Research, 151, 115-123. https://doi.org/10.1016/j.envres.2016.07.026.

Farnesi, L. C., Belinato, T. A., Gesto, J. S. M., Martins, A. J., Bruno, R. V., \& Moreira, L. A. (2019). Embryonic development and egg viability of w Mel-infected Aedes aegypti. Parasites \& Vectors, 12(1), 211. https://doi.org/10.1186/s13071-019-3474-z.

Ferreira, A. C., Chiaravalloti Neto, F., \& Mondini, A. (2018). Dengue in Araraquara, state of São Paulo: epidemiology, climate and Aedes aegypti infestation. Revista de Saúde Pública, 52, 18. https://doi.org/10.11606/S1518-8787.2018052000414.

Kendall, M. G. (1955). Rank correlation methods. Griffin, London.

Kraemer, M. U., Reiner, R. C., Brady, O. J., Messina, J. P., Gilbert, M., Pigott, D. M., YI, D., Johnson, K., Earl, L., Marczak, L. B., Shirude, S., Weaver, N. D., Bisanzio, D., Perkins, T. 
A., Lai, S., Lu, X., Jones, P., Coelho, G. E., Carvalho, R. G., Bortel, W. V., Marsboom, C., Hendrickx, G., Schaffner, F., Moore, C. G., Nax, H. H., Bengtsson, L., Wetter, E., Tatem, A. J., Brownstein, J. S., Smith, D. L., Lambrechts, L., Cauchemez, S., Linard, C., Faria, N. R., Pybus, O.G., Scott, T. W., Liu, Q., Yu, H., Wint, G. R. W., Hay, S. I., \& Golding, N. (2019). Past and future spread of the arbovirus vectors Aedes aegypti and Aedes albopictus. Nature Microbiology, 4(5), 854-863. https://doi.org/10.1038/s41564-019-0376-y.

Lins, T. M., \& Candeias, A. L. B. (2018). Estudo da influência de variáveis socioeconômicas em casos confirmados de Zika em Recife, Pernambuco. Revista Brasileira de Meio Ambiente, $4(1)$.

Marques, C. A., Siqueira, M. M. D., \& Portugal, F. B. (2020). Avaliação da não completude das notificações compulsórias de dengue registradas por município de pequeno porte no Brasil. Ciência \& Saúde Coletiva,25, 891-900. https://doi.org/10.1590/141381232020253.16162018 .

Mayer, S. V., Tesh, R. B., \& Vasilakis, N. (2017). The emergence of arthropod-borne viral diseases: A global prospective on dengue, chikungunya and zika fevers. Acta Tropica, 166, 155-163. https://doi.org/10.1016/j.actatropica.2016.11.020.

Monteiro, E. S. C., Coelho, M. E., Cunha, I. S. D., Cavalcante, M. D. A. S., \& Carvalho, F. A. D. A. (2009). Aspectos epidemiológicos e vetoriais da dengue na cidade de Teresina, PiauíBrasil, 2002 a 2006. Epidemiologia e Serviços de Saúde, 18(4), 365-374. http://dx.doi.org/10.5123/S1679-49742009000400006.

Moura, P. M., Docile, T. N., Arnóbio, A., \& Figueiró, R. (2014). O Desmatamento e o Crescimento urbano desordenado no estado do Rio de Janeiro: impactos na dinâmica do Dengue. Cadernos UniFOA, 9(24), 77-85.

Nachar, N. (2008). The Mann-Whitney U: A test for assessing whether two independent samples come from the same distribution. Tutorials in Quantitative Methods for Psychology, 4(1), 13-20. 
Pettitt, A. N. (1979). A non-parametric approach to the change-point problem. Journal of the Royal Statistical Society: Series C (Applied Statistics), 28(2), 126-135. Doi: https://doi.org/10.2307/2346729.

Pinheiro, A., Graciano, R. L. G., \& Severo, D. L. (2013). Tendência das séries temporais de precipitação da região sul do Brasil. Revista Brasileira de Meteorologia, 28(3), 281-290. https://doi.org/10.1590/S0102-77862013000300005.

Reis, J. L. B., De Lacerda Abrahão, B. O., \& Moura, D. L. (2017). Os problemas do rio São Francisco na percepção de moradores de Petrolina e Juazeiro: a saúde em foco. Saúde e Pesquisa, 10(3), 473-484. https://doi.org/10.17765/2176-9206.2017v10n3p473-484.

Ribeiro, E. A. W. (2019). CHAPECÓ/SC: PARTIAL RESULTS. Hygeia, 15(33), 29-41. http://dx.doi.org/10.14393/Hygeia153351663

Silva, E. B., \& DA Cunha Nóbrega, P. R. (2012). Dengue: reflexões sobre a incidência da doença no município de Palmares, Pernambuco no pós-enchente (2010, 2011). Journal of Management \& Primary Health CarE, 3(2), 106-113. https://doi.org/10.14295/jmphc. v3i2.147.

Sneyres, R. (1990). Technical note no. 143 on the statistical Analysis of Time Series of Observation. World Meteorological Organization, Geneva, Switzerland.

Teixeira, M. G., Siqueira, J. B., Germano JR, L. C., Bricks, L., \& Joint, G. (2013). Epidemiological trends of dengue disease in Brazil (2000-2010): a systematic literature search and analysis. PLoS Neglected Tropical Diseases, 7(12). https://doi.org/10.1371/journal.pntd.0002520.

Valle, D., Pimenta, D. N., \& Da Cunha, R. V. (Eds.). (2015). Dengue: teorias e práticas. SciELO-Editora FIOCRUZ. 
Wilke, A. B. B., Wilk-da-Silva, R., \& MarrellI, M. T. (2017). Microgeographic population structuring of Aedes aegypti (Diptera: Culicidae). PloS One, 12(9), e0185150. https://doi.org/10.1371/journal.pone.0185150.

Yu, P. S., Yang, T. C., \& Kuo, C. C. (2006). Evaluating long-term trends in annual and seasonal precipitation in Taiwan. Water Resources Management, 20(6), 1007-1023. https://doi.org/10.1007/s11269-006-9020-8

Porcentagem de contribuição de cada autor no manuscrito

\author{
Jucarlos Rufino de Freitas - 40\% \\ Edgo Jackson Pinto Santiago - 10\% \\ Juliano Carlo Rufino de Freitas - 10\% \\ Antonio Samuel Alves da Silva - 10\% \\ Renisson Neponuceno de Araújo Filho - 10\% \\ Victor Casimiro Piscoya - 10\% \\ Moacyr Cunha Filho - 10\%
}

